\title{
A Radial Sclerosing Lesion Mimicking Breast Cancer on Mammography in a Young Woman
}

\author{
Masashi Furukawa $^{a}$ Naruto Taira $^{a}$ Shigemichi Iha ${ }^{c}$ \\ Tomohiro Nogami $^{\mathrm{a}}$ Tadahiko Shien $^{\mathrm{a}}$ Masako Omori ${ }^{\mathrm{b}}$ \\ Hiroyoshi Doihara ${ }^{a}$ \\ Departments of ${ }^{\mathrm{a}}$ Breast and Endocrine Surgery and ${ }^{\mathrm{b}}$ Pathology, Okayama \\ University Hospital, and ' Department of Breast Surgery, Okayama Ohfuku \\ Clinic, Okayama, Japan
}

\section{Key Words}

Breast cancer - Radial sclerosing lesion - Mammography · Young women

\begin{abstract}
A spiculated mass on a mammogram is highly suggestive of malignancy. We report the case of a 32-year-old woman with a radial sclerosing lesion that mimicked breast cancer on mammography. She visited her physician after palpating a lump in her left breast. Mammography showed architectural distortion in the upper inner quadrant of the left breast. Ultrasonography showed a low echoic area with an ambiguous boundary. Core needle biopsy was performed because of the suspicion of malignancy. Histological examination did not reveal any malignant cells. After 6 months, the breast lump became larger and the patient was referred to our hospital. Mammography performed in our hospital showed a spiculated mass, and therefore mammotome biopsy was performed. Histological examination revealed dense fibroelastic stroma with a wide variety of mastopathic changes, leading to a diagnosis of a radial sclerosing lesion. One year after the biopsy, the lump on her left breast had disappeared and mammography showed no spiculated mass.
\end{abstract}

\section{Introduction}

Mammography is the most sensitive method for detecting breast lesions, but it lacks specificity. Benign and malignant breast lesions often present with overlapping mammographic findings, and thus a definite radiologic diagnosis may be difficult to 
establish [1]. Spiculated breast lesions, which reflect a wide variety of pathologic entities, are detected relatively frequently on routine mammograms. Here, we report the case of a young woman in whom a radial sclerosing lesion mimicking breast cancer was detected on mammography.

\section{Case Presentation}

A 32-year-old woman visited her physician after she had palpated a lump in her left breast. Mammography showed architectural distortion in the upper inner quadrant of the left breast. Ultrasonography showed a low echoic area with an ambiguous boundary (fig. 1). A core needle biopsy was performed because of suspicion of malignancy. Histological examination did not reveal any malignant cells. Six months after the core needle biopsy, the breast lump became larger and the patient was referred to our hospital. Mammography performed in our hospital showed architectural distortion in the upper inner quadrant of the left breast in a medial lateral oblique view (fig. $2 \mathrm{a}$ ) and a spiculated mass on the craniocaudal spot in a compression magnification view (fig. 2b). Mammotome biopsy was performed because of a strong suspicion of malignancy. Histological examination of the mammotome biopsy revealed a sclerotic area consisting of dense collagenous tissue and entrapped benign ducts. A wide variety of mastopathic changes such as duct hyperplasia, apocrine cysts, sclerosing adenosis, and microcystic deletion of glands were also seen. These findings led to diagnosis of a radial sclerosing lesion (fig. 3 ). One year after the mammotome biopsy, the lump on her left breast had disappeared and mammography showed no spiculated mass (fig. 2c).

\section{Discussion}

The Breast Imaging Reporting and Data System (BI-RADS) developed by the American College of Radiology provides a standardized classification for mammographic studies. Mammograms in BI-RADS category 5 indicate a strong suspicion of malignancy. The specific mammographic features with the highest positive predictive value for malignancy include masses with spiculated margins and/or irregular shapes, as well as calcifications with a linear morphology and/or segmental distribution [2]. The positive predictive value of a biopsy positive for malignancy is 95$97 \%$ for category 5 mammograms $[2,3]$.

Many benign breast lesions, such as abscess, hematoma, radial sclerosing lesion, postsurgical scar, diabetic mastopathy, focal fibrosis, sclerosing adenosis, granular cell tumor, extra-abdominal desmoid tumor, and medial insertion of pectoralis and sternalis muscles, pose diagnostic challenges [1, 4]. Radial sclerosing lesions are benign pseudo-infiltrative lesions characterized by a central zone of fibroelastosis from which epithelial structures radiate out in a stellate formation [5, 6]. The investigation and treatment of these lesions can be difficult because they resemble infiltrating carcinomas radiologically, as well as histologically, due to their similarity to welldifferentiated carcinomas [5-7]. Radial sclerosing lesions show the following characteristics on mammograms: (a) varying appearance in different projections, with no dense solid center, rather than a translucent center; (b) longer and thinner radiating spicules; (c) radiolucent linear structures parallel to spicules, and (d) absence of a palpable lesion or skin changes [6]. The association of these lesions with significant proliferative changes and, in particular, with preneoplastic conditions is becoming increasingly apparent $[5,8,9]$. 
Mammotome biopsy is the method of choice for evaluation of suspicious lesions, since it has been proven to be safe without significant complications, to be easy to perform with high adaptability, and to have excellent patient acceptance. With the advent of the mammotome method many breast lesions can be removed in a breast radiology department, and further excision can be performed if histology shows associated ductal carcinoma in situ or invasive cancer [10]. In our case, the tumor shrank and disappeared on a mammogram 1 year after mammotome biopsy; however, the patient should still be followed up carefully in this type of case. Although a spiculated mass on a mammogram is highly suggestive of breast cancer, our case indicates that a radial sclerosing lesion should be considered as a differential diagnosis.

\section{Disclosure Statement}

The authors have no conflicts of interest to disclose.

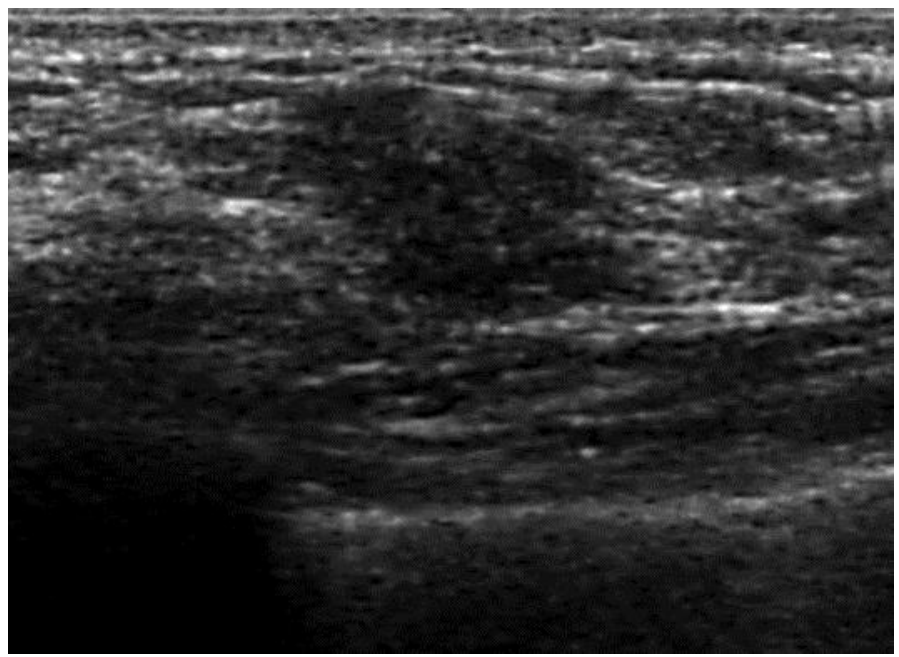

Fig. 1. Ultrasonogram showing a low echoic area with an ambiguous boundary. 

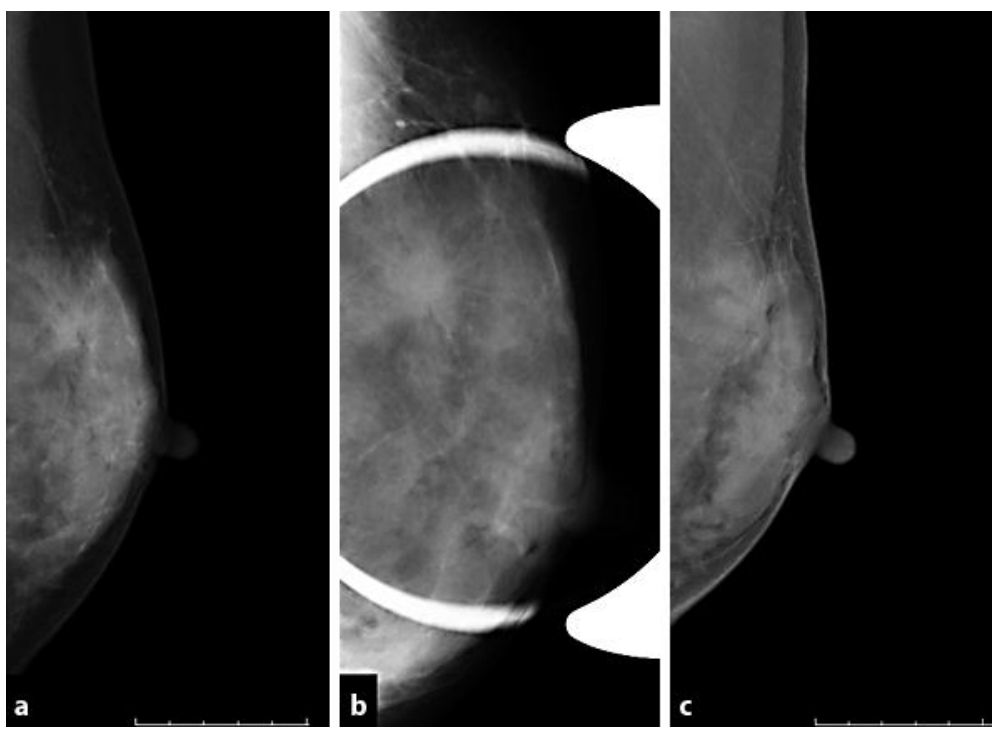

Fig. 2. Mammographic findings. Before mammotome biopsy, architectural distortion in the upper inner quadrant of the left breast was evident in a medial lateral oblique view (a) and a spiculated mass was seen on the craniocaudal spot in a compression magnification view (b). One year after mammotome biopsy, the spiculated mass had disappeared (c).

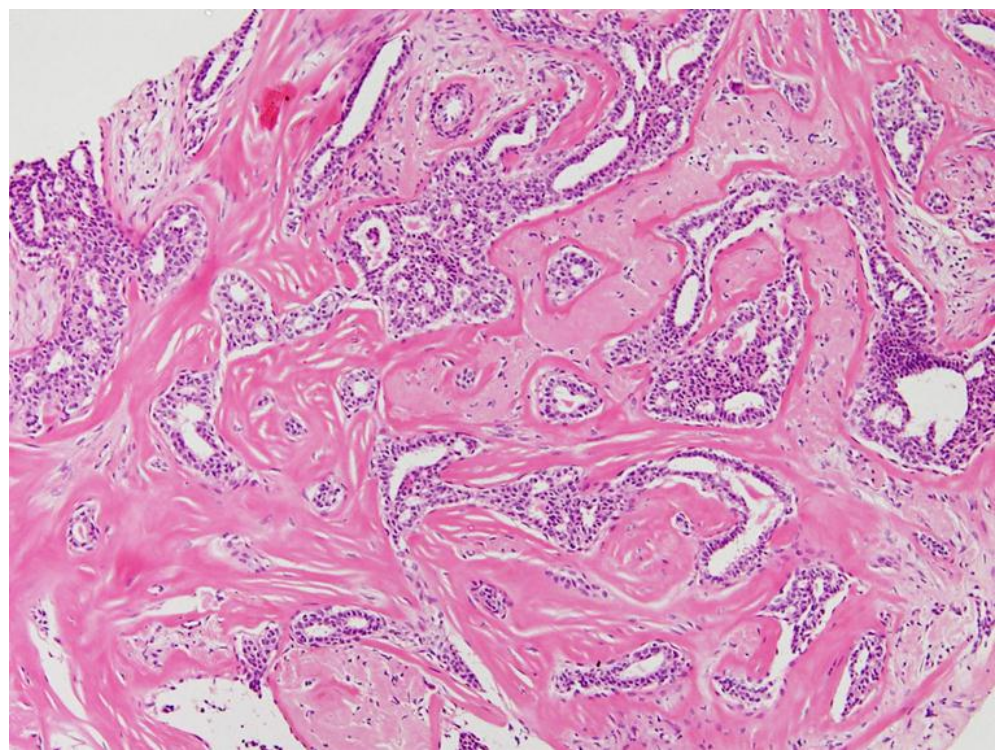

Fig. 3. Mammotome biopsy showing fibroelastic stroma with entrapped small ducts and duct hyperplasia. 


\section{References}

1 Franquet T, De Miguel C, Cozcolluela R, Donoso L: Spiculated lesions of the breast: mammographicpathologic correlation. Radiographics 1993;13:841-852.

-2 Eberl MM, Fox CH, Edge SB, Carter CA, Mahoney MC: BI-RADS classification for management of abnormal mammograms. J Am Board Fam Med 2006;19:161-164.

3 Orel SG, Kay N, Reynolds C, Sullivan DC: BI-RADS categorization as a predictor of malignancy. Radiology 1999;211:845-850.

-4 Pojchamarnwiputh S, Muttarak M, Na-Chiangmai W, Chaiwun B: Benign breast lesions mimicking carcinoma at mammography. Singapore Med J 2007;48:958-968.

5 Fasih T, Jain M, Shrimankar J, Staunton M, Hubbard J, Griffith CD: All radial scars/complex sclerosing lesions seen on breast screening mammograms should be excised. Eur J Surg Oncol 2005;31:1125-1128.

-6 Mitnick JS, Vazquez MF, Harris MN, Roses DF: Differentiation of radial scar from scirrhous carcinoma of the breast: mammographic-pathologic correlation. Radiology 1989;173:697-700.

>7 Grunwald S, Heyer H, Kuhl A, Schwesinger G, Schimming A, Kohler G, Ohlinger R: Radial scar/complex sclerosing lesion of the breast - value of ultrasound. Ultraschall Med 2007;28:206-211.

$>8$ Cawson JN, Malara F, Kavanagh A, Hill P, Balasubramanium G, Henderson M: Fourteen-gauge needle core biopsy of mammographically evident radial scars: is excision necessary? Cancer 2003;97:345-351.

9 Sloane JP, Mayers MM: Carcinoma and atypical hyperplasia in radial scars and complex sclerosing lesions: importance of lesion size and patient age. Histopathology 1993;23:225-231.

10 Mariotti C, Feliciotti F, Baldarelli M, Serri L, Santinelli A, Fabris G, Baccarini M, Maggi S, Angelini L, De Marco M, Lezoche E: Digital stereotactic biopsies for nonpalpable breast lesion. Surg Endosc 2003;17:911-917. 